\title{
Fatty acid biosynthesis in novel ufa mutants of Neurospora crassa
}

\author{
Marta Goodrich-Tanrikulu, Allan E. Stafford, Jiann-Tsyh Lin, \\ Martina I. Makapugay, Glenn Fuller and Thomas A. McKeon
}

Author for correspondence: Marta Goodrich-Tanrikulu. Tel: +1 510559 5627. Fax : +1 5105595777.

United States Department of Agriculture, Agricultural Research Service, Western Regional Research Center, 800 Buchanan Street, Albany, California 94710 , USA

\begin{abstract}
New mutants of Neurospora crassa having the ufa phenotype have been isolated. Two of these mutants, like previously identified ufa mutants, require an unsaturated fatty acid for growth and are almost completely blocked in the de novo synthesis of unsaturated fatty acids. The new mutations map to a different chromosomal location than previously characterized ufa mutations. This implies that at least one additional genetic locus controls the synthesis of unsaturated fatty acids in Neurospora.
\end{abstract}

Keywords: Neurospora crassa, fatty acid desaturation, lipids, oleic acid

\section{INTRODUCTION}

The fatty acid components of membrane and storage lipids are primary determinants of lipid properties. Both fatty acid chain length and degree of unsaturation affect the packing structure, as well as interactions with other lipids and with water, thus determining such properties as membrane fluidity and the melting temperature of triglyceride-based oils. Sixteen- and 18-carbon fatty acids having different degrees of unsaturation are phylogenetically widespread and occur in both membrane lipids, i.e. phospholipids and glycolipids, and in triglycerides. The most widely studied biosynthetic pathway of the 18-carbon unsaturated fatty acids begins with stearate $(18: 0)$, which is desaturated first to oleate $\left(18: 1 \Delta^{9}\right)$, then successively to the polyunsaturates $\alpha$ linoleate $\left(18: 2 \Delta^{9,12}\right)$ and $\alpha$-linolenate $\left(18: 3 \Delta^{9,12,15}\right)$. Mammals, which are unable to synthesize $\alpha$-linoleate or $\alpha$ linolenate, require these fatty acids in the diet. Only plants and certain micro-organisms (cyanobacteria, algae, some bacteria, fungi and protozoa) are able to complete the de novo synthesis of $\alpha$-linolenic acid (Fulco, 1977; Heinz, 1993).

Mutants in biochemical pathways have proven invaluable for understanding the regulation of the pathways and, more recently, for gene isolation. The stearoyl CoA desaturase gene from the yeast Saccharomyces cerevisiae was isolated by complementation of a mutant (ole1) blocked in the synthesis of oleate (Stukey et al., 1990). Saccharomyces,

Abbreviations: DG, diacylglyceride; FAME, fatty acid methyl ester; PC, phosphatidylcholine; $\mathrm{PE}$, phosphatidylethanolamine; TG, triacylglyceride; $16: 0$, palmitic acid; $18: 0$, stearic acid; $18: 1$, oleic acid; $18: 2, \alpha$-linoleic acid; $18: 3, \alpha$-linolenic acid. however, does not synthesize polyunsaturated fatty acids. The filamentous fungus Neurospora crassa is both well characterized genetically and has the ability to synthesize each of the fatty acids in the desaturation pathway leading to $\alpha$-linolenate. Mutants affecting the synthesis of membrane lipids, such as phospholipids and sterols (Scarborough \& Nyc, 1967; Grindle, 1973), and the elongation and desaturation of fatty acids (Lein \& Lein, 1949; Henry \& Keith, 1971; Scott, 1977a) have been isolated and characterized. The previously described desaturation mutants, designated $u f a$, require oleic acid or another unsaturated fatty acid for growth, and lack the ability to synthesize all unsaturated fatty acids. Stocks of the original ufa mutants (Lein \& Lein, 1949) have been lost (Scott, 1977a); later isolates (Scott, 1977a), whose fatty acid synthesis abilities were characterized (Scott, 1977b), have also been lost (Perkins \& Pollard, 1987). Another ufa mutant, presumably a reisolate from the same locus (Perkins \& Pollard, 1987), is available (Fungal Genetics Stock Center). Mutants defective in the desaturation of oleate or linoleate have not been described.

Mutants defective in the synthesis of linoleic acid have been isolated from the cyanobacterium Synechocystis PCC6803 (Wada \& Murata, 1989) by exploiting the ability of cold treatment to increase the endogenous level of and the demand for polyunsaturated fatty acids. Using a requirement of linolenic acid for growth at cold temperatures, we have similarly isolated several Neurospora mutants with altered synthesis of unsaturated fatty acids. Included among these are two mutants with the ufa phenotype, which map to a different chromosomal location than that of the strain currently designated $u f a$. Here, we provide comparative information on fatty acid composition, synthesis and desaturation in these strains 
under conditions which will allow comparison with other mutants affecting the desaturation pathway.

\section{METHODS}

Fatty acid enrichment. Unsaturated fatty acids for routine culture medium supplementation were purified from saponified oils (high-oleic safflower and flax seed oils) by the standard method of urea fractionation (Gunstone, 1967). The fatty acid composition of the enriched fractions was established by GLC of the methyl esters (see below). The oleic acid was $96 \%$ pure; the $\alpha$-linolenic acid was $87 \%$ pure, with the remainder $\alpha$-linoleic acid.

Cultures, media and crosses. The wild-type $N$. crassa strain 74OR23-1A (wt74A), the $u f a$ strain isolated by David D. Perkins and strains for genetic mapping were obtained from the Fungal Genetics Stock Center, Department of Microbiology, University of Kansas Medical Center, Kansas City, KS, USA. The $a^{m 1} a d-3 B c y b-1$ strain was a gift of David D. Perkins.

Liquid culture minimal medium was Vogel's Medium N (VMN) (Davis \& deSerres, 1970) with $1.5 \%$ (w/v) sucrose. Plating medium for colony growth was synthetic crossing medium with $0.01 \%(\mathrm{w} / \mathrm{v})$ glucose and $1 \%(\mathrm{w} / \mathrm{v})$ sorbose in place of sucrose (Davis \& deSerres, 1970). Fatty acid supplementation for all media was $1 \mathrm{mM}$ oleic or $\alpha$-linolenic acid with $1 \%(\mathrm{v} / \mathrm{v})$ of the detergent Tergitol NP40.

Heterokaryon tests for allelism (Davis \& deSerres, 1970) were on solid VMN. Crosses for preliminary genetic mapping were to the linkage tester strain alcoy;csp. Crosses were made by inoculating alcoy; csp as female parent onto slants of synthetic crossing medium supplemented with $1 \%$ Tergitol NP40 and $5 \mathrm{mM}$ oleic acid. Mutant conidia were applied as heterokaryons with the mating helper strain $a^{m 1} a d-3 B c y b-1$ (Perkins, 1984). Progeny ascospores were scored on unsupplemented VMN as described by Davis \& deSerres (1970).

Mutagenesis, selection and screening of unsaturated fatty acid mutants. Wild-type conidia, suspended in tubes of sterile: water at a density of $2 \times 10^{7} \mathrm{ml}^{-1}$, were mutagenized by exposure to $\gamma$ irradiation $\left({ }^{60} \mathrm{Co}\right.$ source) for $4 \mathrm{~h}$ at $10 \mathrm{krad} \mathrm{h}^{-1}$. Irradiated conidia were added to unsupplemented liquid VMN and enriched for mutants by filtration concentration (Davis \&: deSerres, 1970 ) at $15^{\circ} \mathrm{C}$. Remaining ungerminated conidia were plated with supplemental linolenic acid and incubated at $15^{\circ} \mathrm{C}$. Colonies appearing were spot-tested on pairs of gridded plates, one supplemented with linolenic acid, the other with Tergitol NP40 alone. Lines growing only in the presence of linolenic acid were identified as potential mutants in the synthesis of ursaturated fatty acids.

Lines which reproducibly required unsaturated fatty acid supplementation (two additional transfers) were further screened for total fatty acid biosynthetic profiles by radiolabelling and analysis as detailed below.

In some subsequent analyses, radiolabel incorporation patterns of identified mutant lines resembled wild type, indicating uptake of label by revertants. To reduce this problem, one of two methods was used prior to radiolabelling: either cultures were inoculated directly from silica stocks, or conidia were first reenriched for mutants by filtration as above, and treatment with nystatin (Macdonald, 1968). Mutants were treated with $50 \mathrm{U}$ nystatin $\mathrm{ml}^{-1}$ for $3.5 \mathrm{~h}$ at room temperature, filtered, resuspended in supplemented medium, and allowed to recover for $18 \mathrm{~h}$.

Radiolabelling and lipid extraction. Putative mutants with defects in fatty acid desaturation were screened by incubating them with $37 \mathrm{kBq}\left[{ }^{14} \mathrm{C}\right]$ acetate (sodium salt, ICN, 1.48-2.22 $\mathrm{GBq} \mathrm{mmol}^{-1}$ ). Label was added to $25 \mathrm{ml}$ liquid-grown cultures after $1-2 \mathrm{~d}$ of growth at $15^{\circ} \mathrm{C}$. Cultures were harvested by filtration $24 \mathrm{~h}$ after radiolabel addition. The filters plus mycelia were transferred to hot $\left(70^{\circ} \mathrm{C}\right)$ isopropyl alcohol to inactivate lipases (Kates, 1986). To complete the lipid extraction, the isopropyl alcohol was evaporated under $\mathrm{N}_{2}$, and the mycelia reextracted with $5 \mathrm{ml}$ chloroform/methanol/water $[5: 10: 4$, by vol.; $0 \cdot 25 \%$ (v/v) conc. $\mathrm{HCl}$. Chloroform and $\mathrm{CaCl}_{2}$ (final concentration $1 \mathrm{mM})(1.5 \mathrm{ml}$ each) were added to effect a phase separation, and the lower (chloroform) phase was evaporated under $\mathrm{N}_{2}$. For these samples, the lipids were not further fractionated. Instead, fatty acid methyl esters (FAMEs) of the total extract were prepared for radio-HPLC as described below. Mutants identified by this screening method as defective in the synthesis of all unsaturated fatty acids were selected for further study.

For more detailed analysis of the lipid biosynthetic profiles of the ufa mutants, cultures were grown as above with the following modifications. Cultures were incubated at $23^{\circ} \mathrm{C}$ and label was added after the extent of mycelial growth was similar to that of the wild type $18 \mathrm{~h}$ after inoculation. Cultures were radiolabelled with either $370 \mathrm{MBq}\left[{ }^{14} \mathrm{C}\right]$ acetate, or $37 \mathrm{MBq}$ $\left[{ }^{14} \mathrm{C}\right]$ oleate (ICN, $1 \cdot 11-2 \cdot 22 \mathrm{GBq} \mathrm{mmol}^{-1}$ ). Cultures were harvested and extracted $24 \mathrm{~h}$ later as above, and the extracts fractionated as described below.

Chromatographic separation and analysis of lipids. The lipid extracts were dissolved in $2 \mathrm{ml}$ chloroform/acetic acid (100:1, v/v) and fractionated on Sep-Pak silica columns (Waters Assoc.) into neutral lipid, glycolipid and phospholipid fractions by the method of Lynch \& Steponkus (1987). Fractions were split into two tubes, evaporated and redissolved in chloroform/methanol $(1: 1, v / v)$. Half of each fraction was used for analysis of glycerolipid fatty acid composition, and half for identification or autoradiography with Kodak X-Omat AR film. Neutral lipids were separated by TLC on silica gel (EM Science 5715) plates with a two-step development: first development two-thirds up the plate in the solvent system chloroform/ methanol/ethanol/glacial acetic acid $(98: 2: 1: 0 \cdot 1$, by vol.), followed by development in hexane/diethyl ether $(47: 3, \mathrm{v} / \mathrm{v})$. Glycolipids were separated by TLC in the solvent system chloroform/methanol/conc. ammonia (13:5:1, by vol.). Phospholipids were separated by TLC on plates which were first immersed in $100 \mathrm{mM}$ ammonium sulfate and dried for $1.5 \mathrm{~h}$ at $120^{\circ} \mathrm{C}$. Phospholipids were applied to the plates in a spotting chamber. Separation was in the solvent system 1-propanol/ chloroform/propanoic acid/0.1\%(w/v) $\mathrm{KCl}(3: 2: 2: 1$, by vol.). Bands containing lipids to be analysed [i.e. triacylglyceride (TG), 1,2-diacylglyceride (DG), phosphatidylcholine (PC) and phosphatidylethanolamine (PE)] were routinely localized by iodine staining. Lipids were identified by comigration with standards in these and other solvent systems, and by specific spray reagents (Kates, 1986).

FAMEs were prepared by adding $5 \%(\mathrm{v} / \mathrm{v})$ conc. $\mathrm{HCl}$ in methanol to the lipid residue or scraped band and heating in teflon-capped tubes for $1 \mathrm{~h}$ at $100^{\circ} \mathrm{C}$. Methyl esters were extracted three times with hexane.

For radio-HPLC analysis, the hexane was evaporated and the FAMEs redissolved in methanol. Separation of FAMEs was on a Waters Assoc. liquid chromatograph, which consisted of two pumps (M510), a multiwavelength detector (M490), and a data and chromatography control station (M840). The injector was a Rheodyne Model 7125. Separation was by reversed-phase chromatography using a C18 column (Beckman, $5 \mu \mathrm{m}$, Ultrasphere ODS packing, $25 \mathrm{~cm}$ by $4.6 \mathrm{~mm}$ i.d.); the eluent was $90 \%(\mathrm{v} / \mathrm{v})$ methanol at a flow rate of $1.5 \mathrm{ml} \mathrm{min}^{-1}$. Label 
incorporation was measured by a Packard Flo-One/Beta radioactive flow detector. FAMEs were identified by retention times of standards, run previously and detected by UV (205 nm).

FAMEs dissolved in hexane were also analysed for weight $\%$ composition by GLC. FAMEs in the mixtures were identified with a Hewlett-Packard 5880 gas chromatograph equipped with a flame ionization detector and capillary injector system (Hewlett-Packard model 18835B). A fused silica capillary column $(30 \mathrm{~m}$ by $0.25 \mathrm{~mm}$ ) coated with Stabilwax CW20M ( $d f=0.25 \mu \mathrm{m}$; Restek) was used. Detector and injector temperatures were $280^{\circ} \mathrm{C}$ and $260^{\circ} \mathrm{C}$, respectively. The oven temperature was programmed as follows: $80^{\circ} \mathrm{C}$ for $1 \mathrm{~min}$, then

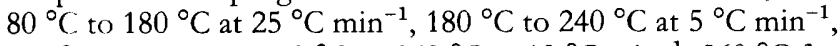
$240^{\circ} \mathrm{C}$ for $10 \mathrm{~min}, 240^{\circ} \mathrm{C}$ to $260^{\circ} \mathrm{C}$ at $10^{\circ} \mathrm{C} \mathrm{m^{-1 }}, 260^{\circ} \mathrm{C}$ for $3 \mathrm{~min}$. Helium was the carrier gas, with splitless injection.

FAMEs were identified by retention times relative to standards and by mass spectrometry with a Hewlett-Packard 5970 quadrupole-based mass selective detector coupled to a HewlettPackard 5790 gas chromatograph. A fused silica capillary column $(25 \mathrm{~m}$ by $0 \cdot 2 \mu \mathrm{m})$ coated with dimethyl silicone ( $d f=0 \cdot 1 \mu \mathrm{m} ; \mathrm{HP}-1$ column) was used to separate FAMEs before introduction into the mass selective detector through a direct capillary interface. Helium was used as carrier gas $(1 \mathrm{ml}$ $\mathrm{min}^{-1}$ ) with splitless injection. The initial oven temperature was $60^{\circ} \mathrm{C}$ for $1 \mathrm{~min}$, then increased to $140^{\circ} \mathrm{C}$ at $25^{\circ} \mathrm{C} \mathrm{min}^{-1}$, next to $220^{\circ} \mathrm{C}$ at $3^{\circ} \mathrm{C} \mathrm{min}^{-1}$, and finally to $280^{\circ} \mathrm{C}$ at $5^{\circ} \mathrm{C} \mathrm{min}{ }^{-1}$. The injector temperature was $260^{\circ} \mathrm{C}$, and the mass selective detector interface was $280^{\circ} \mathrm{C}$. The mass selective detector was operated mainly in the scan mode with the selected ion monitoring mode reserved for trace amounts of FAMEs. Ionization voltage was fixed at $70 \mathrm{eV}$, with the ion source operated at the fixed nominal temperature of $250^{\circ} \mathrm{C}$. Identification of peaks was based on comparison to known spectra and retention times.

\section{RESULTS AND DISCUSSION}

\section{Mutant selection and genetic mapping}

Following $\gamma$ irradiation and filtration enrichment, 17400 colonies were screened for growth in the presence and absence of linolenic acid. Of these, 36 showed a requirement for unsaturated fatty acid supplementation and were further screened for ability to synthesize fatty acids de novo from $\left[{ }^{14} \mathrm{C}\right]$ acetate. Based on this assay, 7 of the 36 mutants were blocked in the synthesis of all unsaturated fatty acids. Each of the mutants was unstable, frequently reverting upon subculturing. Of the seven mutants, five showed extremely slow growth, failed to conidiate, and were infertile in attempted crosses. Growth was not improved by supplying oleate, mixtures of unsaturated fatty acids, or oleate esters (Tween 80 ). The other two mutants, designated GL6 and EH12, grew more slowly than wild type, and on solid medium formed only sparse conidia near the surface of the culture when supplemented with linolenate. Growth was improved when cultures were supplemented with oleate. Cultures most closely resembled the wild type in growth rate and appearance when supplemented with Tween $80(1 \%, \mathrm{v} / \mathrm{v})$. Both mutants mapped to linkage groups I/II in crosses with alcoy; csp. In contrast, the ufa mutant, which shows both a similar growth response and $\left[{ }^{14} \mathrm{C}\right]$ acetate incorporation pattern, mapped to linkage groups IV/V, in agreement with its published map location on IVR (Perkins \&
Pollard, 1987). We selected EH12 and GL6 for further study and comparison to ufa.

In heterokaryon tests of allelism, both strains complemented ufa. In the majority of such tests, GL6 and EH12 complemented each other. Therefore, they appear to be mutations at distinct loci, which we propose designating ufa-3 (GL6) and ufa-4 (EH12). However, since some tests were negative, and interpretation of the tests was complicated by spontaneous reversion, it remains possible that they represent different alleles at the same locus.

\section{Fatty acid synthesis and desaturation}

Based on the initial screening, $\left(\left[{ }^{14} \mathrm{C}\right]\right.$ acetate incorporation into total fatty acids at $15^{\circ} \mathrm{C}$ ), the $u f a$-type mutants ( $u f a$, EH12 and GL6) readily synthesize palmitic and stearic acids, but are unable to synthesize oleic acid or the polyunsaturates $\alpha$-linoleic and $\alpha$-linolenic acid. In more detailed studies, we compared the incorporation of ${ }^{14} \mathrm{C}$ from acetate into individual phospholipids, glycolipids and neutral lipids following fractionation and TLC separation of total lipid extracts. To facilitate later comparison with mutants defective in the subsequent desaturation steps, cultures were grown with a linolenic acid $(87 \%)$ supplement at $23{ }^{\circ} \mathrm{C}$.

Phospholipid (five major phosphorus-positive bands) and glycolipid (one major band) incorporation patterns were similar for each of the mutants and the wild type, as indicated by autoradiography (not shown). The neutral lipid incorporation pattern was also similar, except in some experiments the mutants incorporated more label into free fatty acids than did the wild type. The most intensely radiolabelled neutral lipids were generally TG, followed by sterols, sterol esters, DG and free fatty acids. The most intensely labelled phospholipids were PC and PE. Incorporation into glycolipid was less than that into DG. Relative to the incorporation of label into TG, the amount of label incorporated into DG and phospholipids was higher in the mutants (Table 1), and highest for EH12. EH12 also had lower overall incorporation of label

\section{Table 1. Distribution of $\left[{ }^{14} \mathrm{C}\right]$ acetate in major glycerolipids of Neurospora cultures}

Cultures were supplemented with $1 \mathrm{mM}$ linolenic acid; details of culturing and labelling conditions are described in Methods.

\begin{tabular}{|lccccc|}
\hline Strain & $\begin{array}{c}\text { No. of } \\
\text { expts }\end{array}$ & TG & 1,2-DG & PC & PE \\
\hline wt (74A) & 5 & $100^{*}$ & $0 \cdot 8$ & $12 \cdot 1$ & $10 \cdot 9$ \\
ufa & 3 & 100 & $1 \cdot 3$ & $14 \cdot 4$ & $16 \cdot 3$ \\
GL6 & 3 & 100 & $2 \cdot 8$ & $16 \cdot 4$ & $17 \cdot 4$ \\
EH12 & 3 & 100 & $4 \cdot 6$ & $24 \cdot 7$ & $27 \cdot 9$ \\
\hline
\end{tabular}

* Determination was by radio-HPLC of FAMEs following lipid class separation. Values are means following $24 \mathrm{~h}$ of radiolabelling, expressed relative to 100 for TG. Mean c.p.m. in TG, approximately $1 \times 10^{7}$ (see text for exception). 


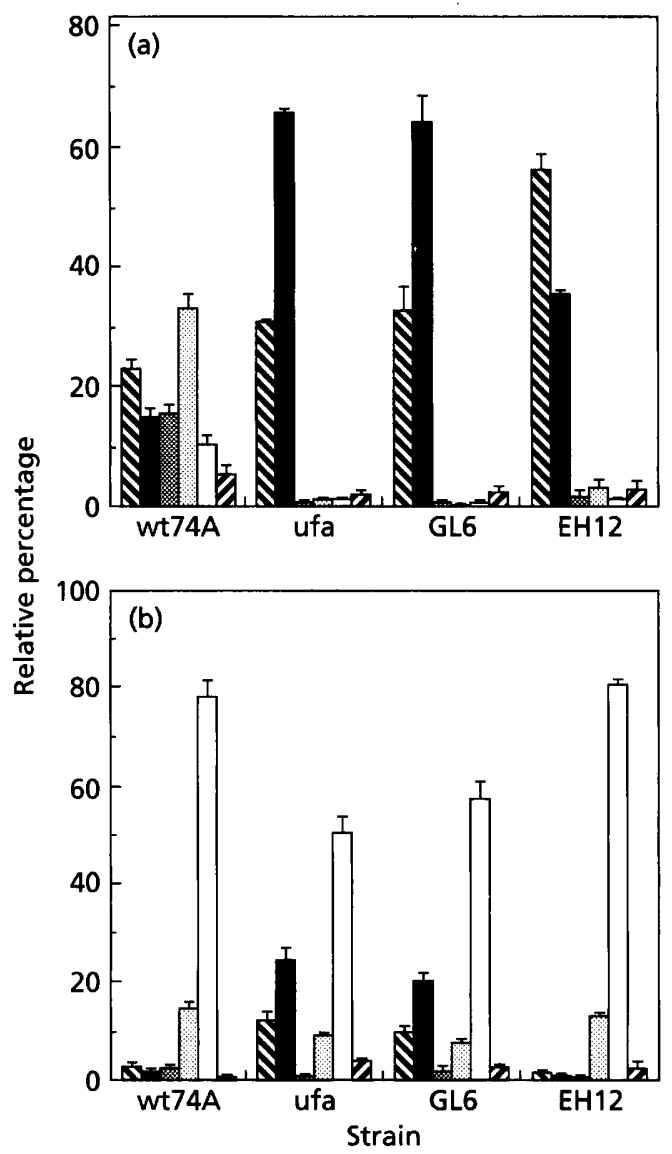

Fig. 1. TG synthesis by 18:3-supplemented Neurospora. (a) $\left[{ }^{14} \mathrm{C}\right]$ Acetate incorporation into TG: relative percentage incorporation into fatty acids of TG $24 \mathrm{~h}$ after label addition to cultures, as described in Methods. Determination was by radioHPLC analysis of FAMEs following lipid class separation. $16: 0 ; \square, 18: 0 ;$, 18:1; $0,18: 2 ; \square, 18: 3$. 'Other' fatty acids (双, unidentified. (b) Relative percentage fatty acid composition of TG by weight, based on GC analysis of FAMEs. 'Other' fatty acids include 14:0, 20:0, 22:0 and 24:0. Values are means of at least three experiments for each strain; error bars indicate SE.

into TG, averaging $28 \%$ of wild type. This is probably because of a slower growth rate, since the amount of TG produced per culture was similarly low.

Comparison of label incorporation into individual fatty acids of TG was consistent with preliminary screening results, and showed clear differences in the incorporation profiles of wild type and mutant cultures (Fig. 1a). Wildtype cultures grown in the presence of linolenic acid at $23^{\circ} \mathrm{C}$ had incorporated most of the $\left[{ }^{14} \mathrm{C}\right]$ acetate label after $24 \mathrm{~h}$ into linoleate and palmitate. The mutant lines, however, incorporated label almost exclusively into palmitate and stearate. The pattern of label incorporation was nearly identical for $u f a$ and GL6, which incorporated most of the label into stearate, whereas EH12 incorporated most into palmitate.

Without supplementation, the amount of linolenic acid in wild-type Neurospora is temperature-dependent; under

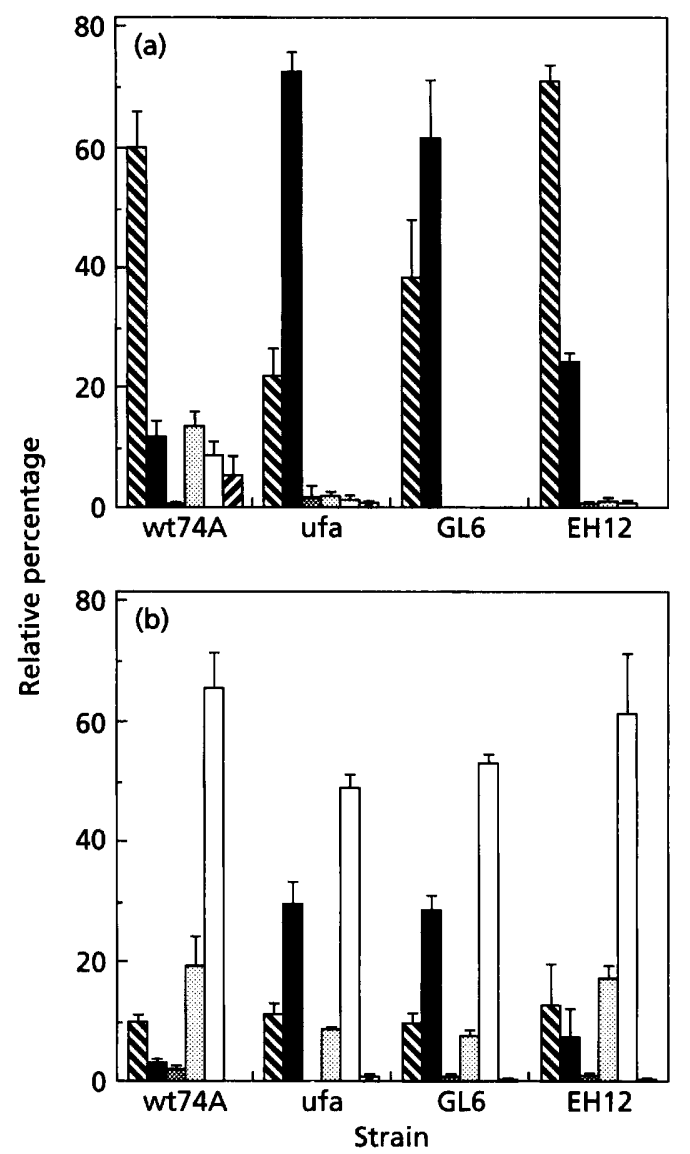

Fig. 2. PC synthesis by $18: 3$-supplemented Neurospora. (a) $\left[{ }^{14} \mathrm{C}\right]$ Acetate incorporation into PC. (b) Relative percentage fatty acid composition of PC by weight. Description as for Fig. 1.

our growing conditions, the wild type accumulates about $8 \%$ linolenic acid into total mycelial lipids at $23{ }^{\circ} \mathrm{C}$. The exogenous linolenic acid was readily taken up by the cultures and incorporated into lipids, especially TG. Fig. 1(b) compares the TG fatty acid composition of the wild type and mutant lines when supplemented with $1 \mathrm{mM}$ linolenic acid. The majority of the fatty acid in the wild type was that of the supplement, which is predominantly linolenic acid (a lesser enrichment for the linoleic acid in the supplement is also evident from Fig. 1b). The $u f a$ and GL6 mutants accumulated high levels of palmitate and stearate, as well as the supplement, into TG. In contrast, the EH12 mutant fatty acid profile more closely resembled that of the wild type. Each of the ufa-type mutants also accumulated about five times the wild-type level of very long-chain fatty acids $(20: 0,22: 0,24: 0)$ (Fig. 1b); the relative ratios of $20: 0$ and $22: 0$ to $24: 0$ fatty acids did not differ from wild type (not shown).

Since phospholipids are important membrane lipids, and PC and DG are also implicated as intermediates in the synthesis of unsaturated fatty-acid-containing TG (Chopra \& Khuller, 1984), we determined the incorporation of acetate-derived label into fatty acids of PC, PE 


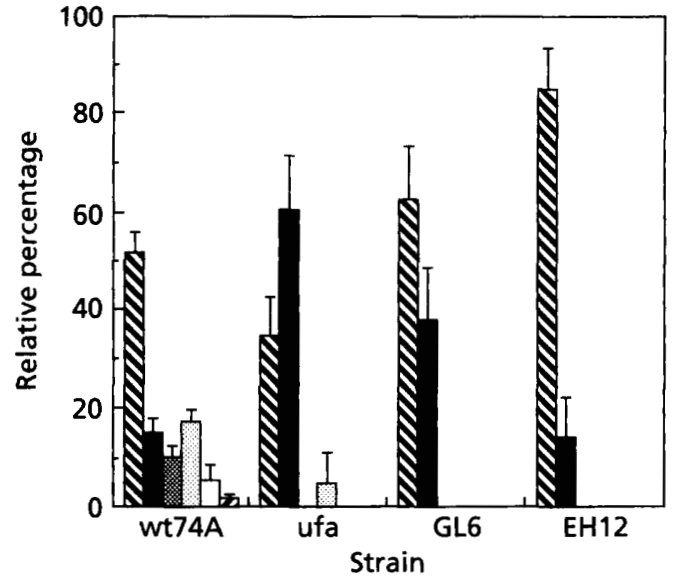

Fig. 3. $\left[{ }^{14} \mathrm{C}\right] \mathrm{Ace}$ tate incorporation into $1,2-\mathrm{DG}$ of $18: 3-$ supplemented Neurospora. Relative percentage incorporation into fatty acids of 1,2-DG was determined by radio-HPLC of FAMEs; details of culturing and labelling conditions are

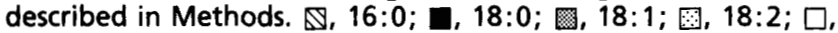
18:3. 'Other' fatty acids ( $\square)$, unidentified. Values are means of two (EH12) or three (wt, ufa, GL6) determinations; error bars indicate SE.

and 1,2-DG. For PC, which is the presumed substrate for oleate and linoleate desaturation, wild-type cultures grown in the presence of linolenic acid had incorporated most of the label after $24 \mathrm{~h}$ into palmitate, and only trace levels into oleate (Fig. 2a), a pattern which differed markedly from that for TG (Fig. 1a). In contrast, the pattern for the mutant lines was similar for PC (Fig. 2a) and TG (Fig. 1a). The EH12 mutant PC incorporation pattern more closely resembled that of the wild type. Comparison of the fatty acid composition of PC (Fig. 2b) among the strains shows similar features to those observed in TG (Fig. 1b); a difference was that palmitate $(16: 0)$ levels in PC were nearly identical for all four strains.

For PE, incorporation profiles were similar to those of PC for each line, except more label was found in palmitate and less in stearate (not shown). Profiles of radiotracer incorporation into 1,2-DG (Fig. 3) indicate a possible difference between the mutants GL6 and ufa. The GL6 mutant averaged more label incorporation than wild type into palmitate, whereas $u f a$ averaged lower incorporation (significant at $90 \%$ ). Again, label incorporation into stearate by EH12 was most similar to the wild type.

In each of the lipid classes examined, the $u f a$-type mutants incorporated trace amounts of label into unsaturated fatty acids (Figs 1a, 2a, 3), which probably indicates a slight phenotypic leakiness, rather than a contribution from a few revertant cells. In some experiments, the TG and label incorporation patterns more closely resembled wild-type patterns than those described above, indicating probable reversion. We did not analyse revertant patterns in detail, but for ufa revertants, the amount of label found in stearate, and its relative percentage, were reduced, and the amount of label in linoleate was increased when compared

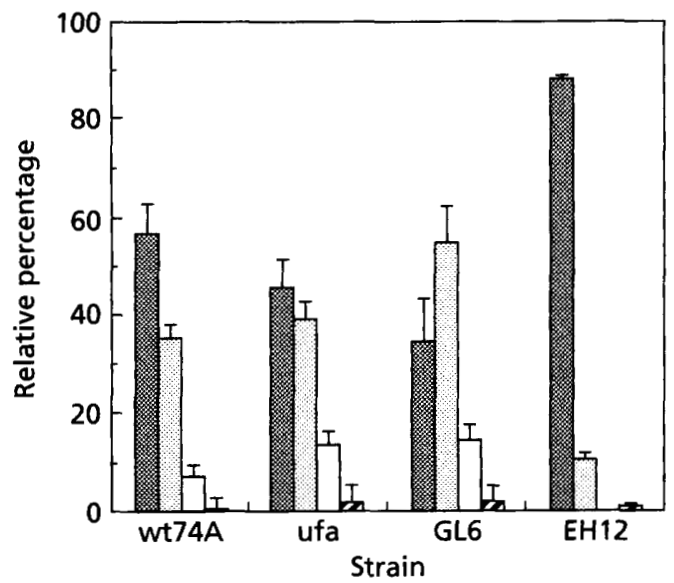

Fig. 4. $\left[{ }^{14} \mathrm{C}\right]$ Oleate incorporation into total fatty acids of 18:3supplemented Neurospora. Determination was by radio-HPLC analysis of FAMEs of total lipids, $24 \mathrm{~h}$ after adding $\left[{ }^{14} \mathrm{C}\right]$ oleate as tracer. $18: 1 ; 0,18: 2 ; \square, 18: 3$. 'Other' fatty acids ( $\square$ ), unidentified. Values are means of two (EH12) or three (wt, ufa, GL6) determinations; error bars indicate SE.

to its typical pattern. Reversion was also usually suspected on observation of a more rapid growth rate.

The mutants lacked the ability to convert stearic to oleic acid. Their ability to desaturate oleate to polyunsaturated fatty acids was tested by labelling total lipids with $\left[{ }^{14} \mathrm{C}\right]$ oleate. The wild type desaturated oleate to $\alpha$-linoleate and to $\alpha$-linolenate. (Fig. 4). The ufa mutant also further desaturated oleate, and produced more labelled linolenate than did the wild type. GL6, like ufa, produced more linolenate than the wild type, and also desaturated more oleate to linoleate. EH12, however, was impaired in the ability to desaturate oleate to linoleate and linoleate to linolenate, since it produced proportionally less at each step than did the wild type. In preliminary characterization, autoradiographic profiles of $\left[{ }^{14} \mathrm{C}\right]$ oleate incorporation into neutral and phospholipids were similar for mutants and wild type (not shown), although oleate incorporation into phospholipids was low for the mutant EH12.

\section{Comparison with previously identified ufa mutants}

In a description of the fatty acid biosynthetic characteristics of the $u f a-1$ and $u f a-2$ mutants, Scott (1977b) noted that the fatty acid supplement used to support their growth affects lipid composition, but still allows differences in composition and desaturation of glycerolipids relative to wild type to be observed. In particular, both neutral and phospholipid composition of supplemented strains are enriched for the unsaturated fatty acid used as supplement, as was observed here (Figs 1a, 2a). Differences in the relative proportion of fatty acids are also affected by temperature (Chopra \& Khuller, 1984). The wild type fatty acid composition which we observed at $23^{\circ} \mathrm{C}$ was lower in palmitate, oleate, and linoleate, and higher in linolenate than reported at $34^{\circ} \mathrm{C}$ 
by Scott (1977b) under similar supplementation conditions.

The previously described $u f a-1$ and $u f a-2$ mutants, like the mutants we studied here, lacked detectable stearate desaturase activity in vivo (Scott, 1977b), and lacked activity in vitro (Gabrielides $e t$ al., 1982), but had the ability to desaturate $\left[{ }^{14} \mathrm{C}\right]$ oleate supplied from the growth medium (Scott, 1977b). Scott also noted that the $u f a-1$ and ufa-2 mutants (except for one ufa-2 allele, TR221) converted more labelled oleate to polyunsaturates than did the wild type. Scott used oleate as unsaturated fatty acid supplement. We observed the same effect in the $u f a$ and GL6 mutants with linolenic acid as supplement (Fig. 4). Several possible explanations could account for the observed higher conversion of labelled oleate by these mutants. One possibility is that the effect observed is one of isotope dilution: oleate pool sizes may be larger in the wild type, as suggested by the higher relative percentage linoleate and linolenate in TG and PC (Figs 1a, 2a). A related possibility is that the oleate synthesized by the wild type in vivo is present in the form of a more readily desaturated lipid, and effectively represents an alternative pool that is preferentially used by Neurospora crassa. A third possibility is that the levels of substrates and products, which differ as mentioned above (Figs 1a, 2a) regulate activity of the desaturases. In wild-type Neurospora, excess substrate stimulates stearate desaturase activity in microsomes, and unsaturated products inhibit it (Gabrielides et al., 1982). In Saccharomyces, similar controls operate, with fatty acid composition affecting OLE1 transcription, OLE1 mRNA levels, and stearate desaturase activity (McDonough et al., 1992). The wild type, as noted, contains a higher percentage of polyunsaturates in PC, and also incorporates proportionally less $\left[{ }^{14} \mathrm{C}\right]$ acetate-derived label into PC than the mutants (Table 1). Each of these explanations could account for the higher level of labelled oleate desaturation observed for $u f a$ and GL6 vs. the wild type.

The third mutant, EH12, which is impaired in all three desaturation steps (Fig. 4), may carry a mutation affecting a component common to each desaturation, for example, a protein participating in electron transport. The reduced level of oleate desaturation by this mutant was similar to that of the earlier exceptional ufa-2 allele TR221 (Scott, 1977b). A similar pleiotropic effect was also noted in a later report of the $u f a-1$ and $u f a-2$ mutants. In addition to lacking stearate desaturase activity, $u f a-1$ was reported to have partial and $u f a-2$ complete absence of oleate desaturase activity in isolated microsomes (Gabrielides et al., 1982). It is not clear why results were different with these mutants when oleate desaturase activities were examined in vivo (Scott, 1977b) and in vitro (Gabrielides $e t$ al., 1982).

The two mutants ufa-1 and ufa- 2 are phenotypically related to those studied here and have been lost (Perkins \& Pollard, 1987), probably as a result of strong selection against the mutants. These mutants both mapped to chromosome IV or V (Perkins et al., 1982). UF $A-1$, represented by a single isolate, appeared to have been a distinct locus from UF $A-2$, based on complementation tests. The $u f a$ mutant is probably a reisolate at one of these loci. The two ufa-type mutants that we have isolated map to a different chromosome (I or II) than does ufa (IVR) (Perkins \& Pollard, 1987), indicating that at least three loci control the synthesis of unsaturated fatty acids in Neurospora. Mutations at any of these loci can abolish the synthesis of unsaturated fatty acids.

The neutral and phospholipid composition (Figs 1b, 2b) and $\left[{ }^{14} \mathrm{C}\right]$ acetate incorporation (Figs $1 \mathrm{a}, 2 \mathrm{a}, 3$ ) profiles of the mutants $u f a$ and GL6 are similar to those of the $u f a-1$ and ufa-2 mutants (Scott, 1977b), relative to wild type. EH12 profiles, in contrast, again are most similar to those of the TR221 allele of $u f a-2$ (Scott, 1977b). Like EH12, some of its profiles were similar to wild type, and in cases where its profile differed from wild type, it still had a profile distinct from that of $u f a-1$ and the other $u f a-2$ alleles. Distinct alleles at any of three (or more) loci can yield the same growth requirement, and yet alter fatty acid profiles in two distinct patterns relative to wild type. Of note, the Saccharomyces ole 1 mutant profiles relative to its wild type (Stukey et al., 1989) most closely resemble those of the ufa, GL6/ufa-1, ufa-2 class, whereas the Aspergillus niger $\mathrm{UFA}_{2}$ mutant profiles (Chattopadhyay et al., 1985) most resemble those of the EH12/TR221 class. Both of these mutants have been assumed to be stearate desaturase mutants. The existence of at least three distinct types of ufa mutants in Neurospora with similar fatty acid biosynthetic profiles raises the question of which mutation is in the structural gene for the stearate desaturase, and what is the biochemical basis of the other mutations.

\section{Essentiality of unsaturated fatty acids for normal growth}

Mutations at these loci lead to a phenotypic requirement of unsaturated fatty acid for growth. For each of the strains, suboptimal supplementation of unsaturated fatty acids caused slowed growth and defective development, including reduced fertility. Mutations eliminating unsaturated fatty acid biosynthesis in other organisms have been reported to have similar effects on growth rate and also to affect mitochondrial function. For example, one of the Aspergillus ufa mutants appears to be a stearate desaturase mutant; other ufa mutants have reduced respiration rates (Chattopadhyay et al., 1985). In $S$. cerevisiae, several unsaturated fatty-acid-requiring (ole) mutants have been isolated. One of these mutations (ole1) is in the structural gene for the stearate desaturase (Stukey et al., 1989); for other ole mutations, the primary defect is in the biosynthesis of ergosterol (Resnick \& Mortimer, 1966) or of cytochromes (Bard et al., 1974), which are involved in the formation of both ergosterol and oleic acid. These unsaturated fatty-acid- or ergosterol-requiring strains are reportedly coisolated at a high frequency with petites (Resnick \& Mortimer, 1966), i.e. strains defective in mitochondrial function. In Saccharomyces, an interdependence of normal mitochondrial function and the synthesis of ergosterol and of oleic acid is known (Parks, 1978). Interestingly, the phenotype of a temperaturesensitive allele of the OLE1 gene, encoding the 
desaturase, shows that it is involved in mitochondrial inheritance (Stewart \& Yaffe, 1991). In plants too, defective growth can be observed: an Arabidopsis thaliana mutant with a high stearate content in leaves and seeds has a dwarf phenotype (Lightner et al., 1993). The evident requirement for a supply of unsaturated fatty acids to allow proper mitochondrial function and to allow normal growth and development probably explains the strong selection pressure for revertants under suboptimal culture conditions.

\section{Implications for selection of TG with desired properties}

Each of the seven Neurospora mutants with the ufa phenotype that we isolated was unstable. We had expected to isolate some non-reverting mutants since mutagenesis was with $\gamma$ irradiation, which can cause deletions. Loss of mutant cultures as a result of reversion has been described for Neurospora ufa mutants (Scott, 1977a; Perkins et al., 1982), the Saccharomyces ole 1 mutant (Stukey et al., 1989) and Apiotricbum curvatum ufa mutants (Ykema et al., 1990). In the case of Saccharomyces, non-reverting ole1 mutants were obtained by disruption of the wild-type OLE1 gene (Stukey et al., 1989). In the case of the oleaginous yeast Apiotrichum, however, each mutant line had a characteristic pattern of reversion, suggesting different loci were affected, and many of the revertants had distinct lipid profiles. The reversion tendency was exploited to derive strains producing TG having different properties; some of the revertants produced TG with properties similar to cocoa butter (Ykema et al., 1990). In soybean, stearate desaturase activity in isolated microsomes was unaffected in mutated lines having high-palmitate (Kopka et al., 1993) or high-stearate (Hui et al., 1993; Kopka \& Jaworski, 1993) seed oil. The Arabidopsis mutation conferring a high-stearate content has four second-site suppressors (Lightner et al., 1993). Desaturation of stearate in diverse organisms thus appears to be affected by mutations at multiple loci, indicating multiple controls for the synthesis of unsaturated fatty acids. A precedent in an animal system is the mammalian stearoyl CoA desaturase, which is regulated by diet, insulin and other factors (Ntambi, 1992).

Programs designed to tailor TG composition for specific end uses by mutant selection or alteration of lipid biosynthetic genes may encounter unexpected problems in line stability, and stable expression of phenotype may require modification of more than one gene. Additionally, undesirable characteristics may accompany the introduction of desired TG compositional traits. For example, high-palmitate lines of Arabidopsis have shown chilling sensitivity under some growing conditions (Wu et al., 1993). Additionally, high-stearate rapeseed lines resulting from anti-sense expression of the stearate desaturase gene have been observed to produce higher levels of very longchain fatty acids (Knutzon et al., 1992), as do the ufa mutants here (Fig. 1b). Neurospora thus serves as an excellent system for examining fatty acid desaturation and may prove useful for preliminary evaluation of genes regulating desaturation in other organisms, such as higher plants.

\section{ACKNOWLEDGEMENTS}

We thank Benjamin E. Gordon at Lawrence Berkeley Laboratories for performing irradiation of conidia and David D. Perkins at Stanford University for advice on crosses.

\section{REFERENCES}

Bard, M., Woods, R. A. \& Haslam, J. M. (1974). Porphyrin mutants of Saccharomyces cerevisiae: correlated lesions in sterol and fatty acid biosynthesis. Biochem Biopbys Res Comm 56, 324-330.

Chattopadhyay, P., Banerjee, S. K., Sen, K. \& Chakrabarti, P. (1985). An unsaturated fatty acid mutant of Aspergillus niger with partially defective $\Delta 9$-desaturase. Can J Microbiol 31, 346-351.

Chopra, A. \& Khuller, G. K. (1984). Lipid metabolism in fungi. CRC Crit Rev Microbiol 11, 209-271.

Davis, R. H. \& deSerres, F. J. (1970). Genetic and microbiological research techniques for Neurospora crassa. Methods Enzymol 17A, 79-143.

Fulco, A. J. (1977). Fatty acid desaturation in microorganisms. In Polyunsaturated Fatty Acids, pp. 19-36. Edited by W.-H. Kunau \& R. T. Holman. Champaign, Illinois: American Oil Chemists' Society.

Gabrielides, C., Hamill, A. L. \& Scott, W. A. (1982). Requirements of $\Delta 9$ and $\Delta 12$ fatty acid desaturation in Neurospora. Biocbim Biophys Acta 712, 505-514.

Grindle, M. (1973). Sterol mutants of Neurospora crassa: their isolation, growth characteristics and resistance to polyene antibiotics. Mol \& Gen Genet 120, 283-290.

Gunstone, F. D. (1967). An Introduction to the Chemistry and Biochemistry of Fatty Acids and their Glycerides, 2nd edn, p. 40. Bungay, Suffolk: Richard Clay.

Heinz, E. (1993). Biosynthesis of polyunsaturated fatty acids. In Lipid Metabolism in Plants, pp. 33-89. Edited by T. S. Moore, Jr. Boca Raton, Florida: CRC Press.

Henry, S. A. \& Keith, A. D. (1971). Saturated fatty acid requirer of Neurospora crassa. J Bacteriol 106, 174-182.

Hui, T., Shanklin, J. \& Nikolau, B. J. (1993). Biochemical characterization of a soybean with high stearic acid seed oil. 1993 Plant Lipid Symposium, Minneapolis: National Plant Lipid Cooperative.

Kates, M. (1986). Techniques of Lipidology. Isolation, Analysis and Identification of Lipids, 2nd edn. Vol. 3, part 2 of Laboratory Techniques in Biochemistry and Molecular Biology. Edited by R. H. Burdon \& P. H. van Knippenberg. Amsterdam, New York, Oxford: Elsevier.

Knutzon, D. S., Thompson, G. A., Radke, S. E., Johnson, W. B. Knauf, V. C. \& Kridl, J. C. (1992). Modification of Brassica seed oil by antisense expression of a stearoyl-acyl carrier protein desaturase gene. Proc Natl Acad Sci US A 89, 2624-2628.

Kopka, J. \& Jaworski, J. G. (1993). Enzyme defects causing a high palmitate and a high stearate soybean phenotype. 1993 Plant Lipid Symposium, Minneapolis: National Plant Lipid Cooperative.

Lein, J. \& Lein, P. S. (1949). Studies on a Neurospora mutant requiring unsaturated fatty acids for growth. J Bacteriol 58, 595-599.

Lightner, J. E., James, D., Dooner, H. \& Browse, J. (1993). High stearate alters growth and development in Arabidopsis: biochemical, genetic and physiological investigation of high 18:0 mutants and their suppressors. 1993 Plant Lipid Symposium, Minneapolis: National Plant Lipid Cooperative. 
Lynch, D. V. \& Steponkus, P. L. (1987). Plasma membrane lipid alterations associated with cold acclimation of winter rye seedlings (Secale cereale L. cv. Puma). Plant Pbysiol 83, 761-767.

Macdonald, K. V. (1968). The selection of auxotrophs of Penicillium chrysogenum with nystatin. Genet Res 11, 327-330.

McDonough, V. M., Stukey, J. E. \& Martin, C. E. (1992). Specificity of unsaturated fatty acid-regulated expression of the Saccharomyces cerevisiae OLE1 gene. J Biol Chem 267, 5931-5936.

Ntambi, J. M. (1992). Dietary regulation of stearoyl-CoA desaturase 1 gene expression in mouse liver. J Biol Chem 267, 10925-10930.

Parks, L. W. (1978). Metabolism of sterols in yeast. CRC Crit Rev Microbiol 6, 301-342.

Perkins, D. D. (1984). Advantages of using the inactive-matingtype $a^{m 1}$ strain as a helper component in heterokaryons. Neurospora Newslett 31, 41-42.

Perkins, D. D. \& Pollard, V. C. (1987). Newly mapped chromosomal loci of Neurospora crassa. Fungal Genet News/ 34, 52-53.

Perkins, D. D., Radford, A., Newmeyer, D. \& Björkman, M. (1982). Chromosomal loci of Neurospora crassa. Microbiol Rev 46, 426-570.

Resnick, M. A. \& Mortimer, R. K. (1966). Unsaturated fatty acid mutants of Saccharomyces cerevisiae. J Bacteriol 92, 597-600.

Scarborough, G. E. \& Nyc, J. F. (1967). Methylation of ethanolamine phosphatides by microsomes from normal and mutant strains of Neurospora crassa. J Biol Chem 242, 238-242.

Scott, W. A. (1977a). Unsaturated fatty acid mutants of Neurospora crassa. J Bacteriol 130, 1144-1148.
Scott, W. A. (1977b). Mutations resulting in an unsaturated fatty acid requirement in Neurospora. Evidence for $\Delta 9$-desaturase defects. Biochemistry 16, 5274-5279.

Stewart, L. C. \& Yaffe, M. P. (1991). A role for unsaturated fatty acids in mitochondrial movement and inheritance. J Cell Biol 115, 1249-1257.

Stukey, J. E., McDonough, V. M. \& Martin, C. E. (1989). Isolation and characterization of OLE1, a gene affecting fatty acid desaturation from Saccharomyces cerevisiae. J Biol Chem 264, 16537-16544.

Stukey, J. E., McDonough, V. M. \& Martin, C. E. (1990). The OLE1 gene of Saccharomyces cerevisiae encodes the $\Delta 9$ fatty acid desaturase and can be functionally replaced by the rat stearoyl-CoA desaturase gene. J Biol Chem 265, $20144-20149$.

Wada, H. \& Murata, N. (1989). Synechocystis PCC6803 mutants defective in desaturation of fatty acids. Plant Cell Pbysiol 30, 971-978.

Wu, J., James, D., Dooner, H. \& Browse, J. (1993). Biochemical and physiological characterization of a mutant of Arabidopsis with increased palmitic acid. 1993 Plant Lipid Symposium, Minneapolis: National Plant Lipid Cooperative.

Ykema, A., Verbree, E. C., Verwoert, I. I. G. S., van der Linden, K. H., Nijkamp, H. J. J. \& Smit, H. (1990). Lipid production of revertants of $u f a$ mutants from the oleaginous yeast Apiotrichum curvatum. Appl Microbiol Biotechnol 33, 176-182.

Received 26 April 1994; accepted 14 June 1994. 